\title{
The Effect of Aromatherapy Inhalation on Reducing Chronic Pain for Children with Cancer: A Pilot Study
}

\author{
Komang Yogi Triana ${ }^{1, *}$, Allenidekania Allenidekania ${ }^{2}$ and Happy Hayati ${ }^{2}$ \\ ${ }^{1}$ Department of Nursing, Sekolah Tinggi Ilmu Kesehatan Bina Usada, Bali 80361, Indonesia \\ ${ }^{2}$ Department of Pediatric Nursing, Faculty of Nursing, Universitas Indonesia, \\ Jawa Barat 16424, Indonesia
}

(*Corresponding author's e-mail: yogitriana25@gmail.com)

Received: 22 September 2020, Revised: 24 May 2021, Accepted: 22 June 2021

\begin{abstract}
Chronic pain is a physical symptom often experienced by pediatric patients with cancer. Longlasting pain conditions can cause the deterioration of the quality of life of children. In order to enhance their quality of life, they should get any intervention to control the pain. Besides the pharmacologic pain management, nurses also should develop a non pharmacologic pain management strategy. One of the simplest and cheap strategy which is currently being developed was aromatherapy. This study aims to determine the effectiveness of aromatherapy for controlling chronic pain experienced by pediatric patients with cancer. The method used is a quasi-experiment with a consecutive sampling technique. There were 20 respondents involved, which were divided randomly into 2 groups. This study showed that inhaled aromatherapy proved effective for reducing chronic pain $(\mathrm{p}=0.001)$ compared with standard care. Aromatherapy also have other positive effects that make the patient feel more relaxed and improve the patient's sleep quality. Thus, the use of inhaled aromatherapy interventions can be considered to use in controlling the chronic pain experienced by pediatric patients with cancer to optimize the nursing care. However, further research is still needed by involving a larger number of respondents.
\end{abstract}

Keywords: Cancer, Chronic pain, Inhaled aromatherapy, Pediatric

\section{Introduction}

Cancer has been reported as one of the leading causes of death in children. Children with cancer experience various unpleasant physical and psychological signs and symptoms [1]. One of the most common symptoms is the physical pain brought on by the development process of a patient's disease [2]. This condition can last a long time - as long as the child still has cancer cells in his/her body [3]. Persistent pain experienced by children can also lead to physical consequences, such as obstacles in physical mobility, decreased immunity, decreased appetite and concentration, sleep disorders and disruption in socializing [4]. Whereas psychologically, the condition of chronic pain is associated with the emergence of Post-Traumatic Stress Symptoms (PTSS) that can emerge when children begin to enter adulthood [5]. The use of medical drugs, such as opioids, is an option for reducing patient pain, but the potential side effects that children may experience are harsh, such as constipation, urinary retention, nausea, sedation and respiratory depression. Therefore, nurses as health professionals are expected to implement specific strategies to control chronic pain felt by patients [6]. This task is important, considering that children with cancer will likely experience long-term pain, and this can reduce their quality of life [7].

Another that most children with chronic pain rarely use analgesics in their daily lives. They prefer to use non-pharmacological pain management techniques, such as ice compresses, warm compresses or massages, to control the pain [8]. One non-pharmacological pain management technique that is currently being widely used is aromatherapy [9]. Aromatherapy is a form of complementary and alternative medicine (CAM) treatment that uses essential extracts from plants, especially herbs and essential oils [10]. Some essential oils used in aromatherapy are believed to have positive effects on the body, one of which has the effect of reducing chronic pain [11]. However, research on the benefits of aromatherapy for pain that felt by children today is still complicated. Some researches shows that the response of pediatric patients to aromatherapy is good by the physiological and psychological aspects. One of them is reduced 
frequency of analgesic use and improved sleep quality. But the other research suggested that there was no difference in pain during the blood draw in children compared to control group. The purpose of this study is to identify the effectiveness of aromatherapy inhalation for controlling chronic pain in pediatric patients with cancer.

\section{Materials and methods}

This study uses a quasi-experimental method with a sample selection technique was consecutive sampling in accordance with the inclusion criteria: School-age children and adolescents ( 7 - 17 years), with a diagnosis of cancer, experiencing chronic pain (for more than 1 month), get moderate analgesics/opioids. The exclusion criteria from the sample include: Pediatrics patient who have olfactory problems, psychological disorders, developmental disorders or decreased consciousness as well as those who use oxygen aids.

The study was conducted at the Cipto Mangunkusumo Hospital in Indonesia especially in the NonInfection Room from February to April 2018. The study involved 20 respondents who has assigned the informed consent, which were randomly selected and divided into intervention group and control group. The respondents who were selected as the intervention group identified a scent they liked, and when they felt pain after analgesic administration (for example after $2 \mathrm{~h}$ ), the nurses who were trained how the procedure of the aromatherapy inhalation would assess the pain using the Visual Analog Scale (VAS) technique and provide an aromatherapy intervention for the respondent by dripping the selected essential oil (as many as 4 drops) on clean gauze and then sticking it to the patient's chest area with a distance is 20 $\mathrm{cm}$. The nurse would then review the patient's pain at 10 and $30 \mathrm{~min}$. In addition, the researchers also conducted an evaluation of the patients' and their parents' views about the perceived changes experienced since using aromatherapy to control pain. The control group only received standard care using pain killers and a relaxing technique.

The data collection instrument that was used in this study to measure a patient's pain scale was the VAS-P which shows a pain scale from 0 - 10 [12]. The pain scale of each group was measured at 0,10 and $30 \mathrm{~min}$ by VAS-P. When the respondent began to complained about his/her pain, then we measured the pain for the minutes 0 , then we gave him/her the aromatherapy intervention. Then after $10 \mathrm{~min}$ he/she ingested the aromatherapy, we measure again the pain scale by VAS-P, and for the last, we measured the pain scale after $30 \mathrm{~min}$ of aromatherapy inhalation. After the data were collected, they were processed using a computer program, and the univariate and bivariate were analysed by conducting data normality tests with the Saphiro Wilk test. A homogeneity test was also carried out with Levene's test, and to see the difference between the 2 groups, an independent unpaired test was performed. This research has passed the ethical review from the Faculty of Nursing, Universitas Indonesia with the number: No. 51/UN2.F12.D/ HKP.02.04/2018.

\section{Results and discussion}

Characteristics of respondents

The results of this study indicated that the average age of the respondents was $12.15 \pm 2.907$ years, with the majority of respondents are male $(55 \%)$. The average duration of pain felt by the respondent was $4.65 \pm 4.19$ months, and the type of cancer diagnosis most frequently involved in this study was Ewing Sarcoma (25\%). The pain-killer medication most commonly used by the patients was Morphine $(65 \%)$, and the most favourite aromatherapy was Aloe Vera (15\%).

Table 1 Pain perceived by children before and after interventions in the control and intervention groups in the non-infection room in RSCM $2018(n=20)$.

\begin{tabular}{lcccc}
\hline \multicolumn{1}{c}{ Pain response } & n & Mean & SD & 95 \% CI \\
\hline Minute 0 & 10 & 4.4 & 0.966 & $3.71-5.09$ \\
Intervention & 10 & 4.2 & 1.317 & $3.26-5.14$ \\
Control & & & & \\
Minute 10 $^{\text {th }}$ & 10 & 2.1 & 1.287 & $1.18-3.02$ \\
Intervention & 10 & 5.0 & 0.667 & $4.52-5.48$ \\
Control & & & & \\
Minute 30 & 10 & 0.4 & 0.966 & $0.29-1.09$ \\
Intervention & 10 & 6.6 & 1.43 & $5.58-7.62$ \\
Control & & & & \\
\hline
\end{tabular}


The difference in the mean scale of pain felt by respondents before and after the intervention was obtained by analyzing the paired difference test paired T-test on normally distributed data and Wilcoxon for data that are not normally distributed. The difference in the mean pain scale was obtained from the mean pain scale of the patients at 0,10 , and $30 \mathrm{~min}$ in the 2 groups, presented in the Table 2.

Table 2 Mean differences of pain scale perceived by respondents at 0,10 and $30 \mathrm{~min}$ in the control and intervention group in the non-infection room in RSCM $2018(\mathrm{n}=20)$.

\begin{tabular}{ccccc}
\hline Pain Scale Variable & Mean & SD & $\mathbf{9 5} \% \mathbf{C I}$ & p-value \\
\hline Pre - Minute 10 & 2.3 & 1.337 & $1.343-3.257$ & 0.000 \\
$\quad$ Intervention & -0.8 & 1.619 & $-1.958-0.358$ & 0.153 \\
$\quad$ Control & & & & \\
Pre - Minute 30 & 4 & 1.563 & $2.882-5.118$ & 0.000 \\
$\quad \begin{array}{l}\text { Intervention } \\
\quad \text { Control }\end{array}$ & -2.4 & 1.578 & $-3.529-(-1.271)$ & 0.001 \\
Minute 10 - 30 & & & & $0.942-2.458$ \\
$\quad$ Intervention & 1.7 & 1.059 & $-2.505-(-0.695)$ & 0.001 \\
$\quad$ Control & -1.6 & 1.265 & & \\
\hline
\end{tabular}

The comparison of the mean difference in the pain scale felt by respondents before and after the intervention in the control and intervention groups was obtained by performing an unpaired difference test analysis (Independent T-test) on normally distributed data, and Mann Whitney for data that were not normally distributed. Comparison of the mean differences in the respondents' pain scale at 0,10 , and 30 min in the 2 groups, is presented in the following table form (Table 3 ).

Table 3 Comparison of the mean difference in pain scale perceived by respondents in the control and intervention groups in the non-infection room in RSCM $2018(\mathrm{n}=20)$.

\begin{tabular}{ccccc}
\hline Variable & Mean & SD & Mean Diff. & p-value \\
\hline Difference in mean of pain in pre-post 10 min & 0.75 & 2.149 & -3.100 & 0.001 \\
Difference in mean of pain in pre-post 30 min & 0.8 & 3.622 & -6.400 & 0.001 \\
Difference in mean of pain in pre-post 10-30 min & 0.05 & 2.038 & -3.300 & 0.001 \\
\hline
\end{tabular}

Based on Tables 1 and 2, it can be concluded that the mean pain scale experienced by respondents in each group experienced changes. Table 2 explained about there is a change in the pain scale felt by each respondent in each time span from $0-10,0-30$, and from $10^{\text {th }}$ to the $30^{\text {th }}$ min, which is the intervention group shown that the mean pain scale of the respondents were decreased, but the mean pain scale in the control group were increased.

Based on Table 3, it is evident that the p-values were $0.001(<0.05)$ as the result of the independent T-test between the control and the intervention group in all 3 measurements, it means that there is a significant difference in the decrease in the pain scale of respondents with chronic pain between the provision of aromatherapy inhalation interventions compared to treatment measures routinely performed in the room ( $\mathrm{p}$-value $=0.001$ ) both in the $10^{\text {th }}$ and $30^{\text {th }}$ min. Table 3 also showed that when compared to other pain differences, the largest pain scale change occurs between 0 and $30 \mathrm{~min}$, with a mean difference reached $-6,400$. Thus, aromatherapy inhalation interventions have proven to be effective in controlling chronic pain in pediatric patients, compared to routine care.

\section{Patient and family reports}

The reports from the intervention group and their parents revealed that they felt more relaxed and calmer using aromatherapy, and they were not focused on the pain, which reduced their experience of the pain. Patients and their parents also said that the use of aromatherapy allowed the patients to sleep more soundly so that they rarely complained of pain, and their medication was taken more regularly as well. Respondents also stated that they wanted to continue using aromatherapy until they back home because they liked it. 


\section{Results and discussion}

The average age of respondents involved in this study was 12 years old, which is accordance with research by previous study that revealed that as many as $84 \%$ of cases of cancer in children are experienced at the age of less than 15 years [13]. The most common type of cancer experienced by the patients in this study was Ewing Sarcoma. Another previous study also revealed that the type of bone cancer that most often occurs in adolescent children is Ewing Sarcoma and Osteosarcoma, with more frequent incidents occurring in boys than in girls. This is also consistent with the results of this study, where the majority of respondents were male $(56 \%)$ [14].

In patients who received aromatherapy intervention, it is evident that there was a significant decrease in pain scale from the $10^{\text {th }}$ to the $30^{\text {th }} \mathrm{min}$, whereas in the control group, which only received routine care, there was an increase in the pain scale that the patient felt. Pathophysiologically, when essential oils containing aromatherapy from plants are inhaled, the receptor cells of the olfactory nerve is stimulated, and impulses are transmitted to the emotional centre of the brain and limbic system. Furthermore, the mixing of the transmitted impulses causes the release of internal body chemicals, including enkephalin and endorphins, which can reduce pain and anxiety as well as the levels of epinephrine and norepinephrine. Thus, aromatherapy can be beneficial for reducing agitation, neuroleptics, anxiety and pain [15].

These findings are in accordance with a previous article that revealed in their research that the use of aromatherapy inhalation is effective for controlling the pain of patients with limb fractures, when compared to those not receiving aromatherapy and only getting analgesics. However, use of aromatherapy did not show significant differences in the patient's vital signs [16]. Another study suggests that aromatherapy interventions can be applied as a CAM in the health care setting [16]. This is consistent with other studies which revealed that aromatherapy, as one of the CAMs, is effective for controlling patient pain and does not cause harmful side effects for its users [17].

In addition, other result shows that aromatherapy interventions are effective for reducing abdominal pain due to dysmenorrhea in patients compared to receiving analgesic drugs. Furthermore, the use of aromatherapy can make patients more relaxed, and the breath frequency of patients tends to be lower than those not using aromatherapy [11]. Aromatherapy effect is also been discussed in a systematic review, which states that aromatherapy is effective for reducing pain in both adult patients and children and is a supportive and affordable action that is easily applied compared to others [18]. Furthermore, there are no harmful side effects, so it is safe to use. These results are in accordance with the statement expressed by the room nurse regarding the implementation of aromatherapy inhalation interventions that are felt to be easily applied and liked by patients [15].

The results of interviews conducted with patients and their patients, suggest that using the aromatherapy helps patients sleep more soundly than before and feel more relaxed. The use of aromatherapy inhalation was effective for improving the sleep quality of patients and was able to reduce depression [10]. In addition, other study also had similar results and suggested that the use of aromatherapy also had other positive effects for patients with cancer, such as reducing depression, reducing anxiety, improving sleep quality and improving patient welfare [16].

The patient's parents said that the patients were less likely to ask for opioid drugs than before because they were able to have more control over the pain they felt. This is consistent with previous result that the use of aromatherapy inhalation interventions was effective for reducing the use of analgesic drugs after the third day in post tonsillectomy patients [19]. Pediatric nurses, as health service providers in hospitals, are expected to facilitate patient comfort in various forms, one of which is providing aromatherapy interventions for cancer patients with chronic pain so that they can better control their pain and feel more relaxed and calmer.

\section{Conclusions}

Aromatherapy inhalation interventions have shown to be effective for controlling chronic pain in pediatric patients with cancer when compared to standard care. The use of aromatherapy also has other positive effects, such as improved sleep quality, feelings of relaxation and reduced frequency of additional analgesic drug use. Its application is easy, inexpensive, safe and preferred by children. Thus, aromatherapy inhalation interventions are one of the CAM options that can be considered to applied to patients with chronic pain to improve patient comfort. However, further research is expected to involve a larger number of samples to obtain more convincing results. 


\section{Acknowledgements}

The study was supported by Universitas Indonesia especially for representative literature resources.

\section{References}

[1] RL Siegel, KD Miller and A Jemal. Cancer statistics. CA Cancer J. Clin. 2018; 68, 7-30.

[2] LMC Batalha, A Fernandes, CD Campos and A Goncalves. Pain assessment in children with cancer: A systematic review. Pain Res. Manag. 2015; 4, 119-27.

[3] M McCarthy, R Glick, J Green, K Plummer, K Peters, L Johnsey and C Deluca. Comfort first: An evaluation of a procedural pain management programme for children with cancer. Psycho-Oncology $2013 ; 22,775-82$.

[4] N Canbulat and AS Kurt. Pain management and nursing approaches in pediatric oncology. In: O Ozdemir (Ed.). Complementary pediatrics, InTech, 2012.

[5] AL Holley, AC Wilson, M Noel and TM Palermo. Post-traumatic stress symptoms in children and adolescents with chronic pain: A topical review of the literature and a proposed framework for future research. Eur. J. Pain 2016; 20, 1371-83.

[6] Y Bao, X Kong, L Yang, R Liu, Z Shi, W Li, B Hua and W Hou. Complementary and alternative medicine for cancer pain: An overview of systematic reviews. J. Evid. Based Complement. Altern. Med. 2014; 2014, 170396.

[7] T Hamner, RD Latzman, NE Latzman, TD Elkin and S Majumdar. Quality of life among pediatric patients with cancer: Contributions of time since diagnosis and parental chronic stress. Pediatr. Blood Cancer 2015; 62, 1232-6.

[8] MA Fortier, A Wahi, C Bruce, EL Maurer and R Stevenson. Pain management at home in children with cancer: A daily diary study. Pediatr. Blood Cancer 2014; 61, 1029-33.

[9] B Katja, B Arndt and O Thomas. Aromatherapy as an adjuvant treatment in cancer care - a descriptive systematic review. Eur. J. Integr. Med. 2012; 4, 129.

[10] M Lee, S Lim, JA Song, ME Kim and MH Hur. The effects of aromatherapy essential oil inhalation on stress, sleep quality and immunity in healthy adults: Randomized controlled trial. Eur. J. Integr. Med. 2017; 12, 79-86.

[11] M Uysal, HY Dogru, E Sapmaz, U Tas, B Cakmak, AZ Ozsoy, F Sahin, S Ayan and M Esen. Investigating the effect of rose essential oil in patients with primary dysmenorrhea. Complement. Ther. Clin. Pract. 2016; 24, 45-9.

[12] MJ Hockenberry and D Wilson. Wong's essentials of pediatric nursing. $7^{\text {th }}$ eds. Mosby Elsevier, St. Louis, 2009.

[13] MS Hossain, M Begum, MM Mian, S Ferdous, S Kabir, HK Sarker, S Karim, S Choudhury, A Khan, ZJ Khan and HE Karim-Kos. Epidemiology of childhood and adolescent cancer in Bangladesh, 2001 - 2014. BMC Cancer 2016; 16, 104.

[14] PC Valery, M Laversanne and F Bray. Bone cancer incidence by morphological subtype: A global assessment. Cancer Causes Control 2015; 26, 1127-39.

[15] TMF Marzouk, AMR El-Nemer and HN Baraka. The effect of aromatherapy abdominal massage on alleviating menstrual pain in nursing students: A prospective randomized cross-over study. Evid. Based Complement. Altern. Med. 2013; 2013, 742421.

[16] P Singh and A Chaturvedi. Complementary and alternative medicine in cancer pain management: A systematic review. Indian J. Palliat. Care 2015; 21, 105-15.

[17] N Sut and H Kahyaoglu-Sut. Effect of aromatherapy massage on pain in primary dysmenorrhea: A meta-analysis. Complement. Ther. Clin. Pract. 2017; 27, 5-10.

[18] SE Lakhan, H Sheafer and D Tepper. The effectiveness of aromatherapy in reducing pain: A systematic review and meta-analysis. Pain Res. Treat. 2016; 2016, 8158693.

[19] R Soltani, S Soheilipour, V Hajhashemi, G Asghari, M Bagheri and M Molavi. Evaluation of the effect of aromatherapy with lavender essential oil on post-tonsillectomy pain in pediatric patients: A randomized controlled trial. Int. J. Pediatr. Otorhinolaryngol. 2013; 77, 1579-81. 